\title{
Complete Response of Synchronous Liver Metastasis in a Pancreatic Ductal Adenocarcinoma: Could Surgery Represent a Therapeutic Option?
}
Antonella Argentiero $^{1 *}$, Oronzo Brunetti ${ }^{1 *}$, Angela Calabrese ${ }^{2}$, Angela Monica Sciacovelli ${ }^{1}$, Sabina Delcuratolo ${ }^{1}$, Antonio Giovanni Solimando ${ }^{1,3^{\circ}}$ \& Nicola Silvestris ${ }^{1,4^{\circ}}$

\footnotetext{
${ }^{1}$ Medical Oncology Unit, National Cancer Research Centre, IRCCS Istituto Tumori "Giovanni Paolo II", Bari, Italy

${ }^{2}$ Radiology Unit, National Cancer Research Centre, IRCCS Istituto Tumori "Giovanni Paolo II", Bari, Italy

${ }^{3}$ Department of Biomedical Sciences and Human Oncology, Section of Internal Medicine "G. Baccelli”, University of Bari Medical School, Bari, Italy

${ }^{4}$ Department of Biomedical Sciences and Human Oncology, Section of Medical Oncology, University of Bari Medical School, Bari, Italy

${ }^{*}$ Co-first authors

${ }^{\circ}$ Co-last authors
}

\section{Corresponding author}

Nicola Silvestris, MD

University of Bari

Piazza Giulio Cesare

70124 - Bari

Italy

E-mail: n.silvestris@oncologico.bari.it 


\begin{abstract}
Metastatic pancreatic ductal adenocarcinoma pancreatic (PDAC) is characterized by poor prognosis and short survival. Today, the use of new polytherapeutic regimens increases clinical outcome of these patients opening new clinical scenario.

A crucial issue related to the actual improvement achieved with these new regimens is represented by the occasional possibility to observe a radiological complete response of metastatic lesions in patients with synchronous primary tumor. What could be the best therapeutic management of these patients? Could surgery represent an indication?

Herein we reported a case of a patient with a PDAC of the head with multiple liver metastasis, who underwent first line chemotherapy with mFOLFIRINOX. After 10 cycles, he achieved a complete radiological response of liver metastases and a partial response of pancreatic lesion. A, duodenocephalopancreasectomy was performed. Due to liver a lung metastases after 8 months from surgery, a second line therapy was started with a disease free survival and overall survival of 8 months and 45 months, respectively.

Improvement in the molecular characterization of PDAC could help in the selection of patients suitable for multimodal treatments.
\end{abstract}

\title{
Key words
}

FOLFIRINOX; pancreatic ductal adenocarcinoma; surgery; liver metastases; long term survival 


\section{Introduction}

Pancreatic ductal adenocarcinoma (PDAC) is one of the most important issues in the context of cancer being the fourth leading cause of death in USA and the sixth in Europe and Japan [1-2] with a 5-year survival no greater than 6\% [2] and an estimated increase in incidence that will bring it to the second leading cause of cancer death in 2030 [3]. At first diagnosis, only 10\%-20\% of PDAC patients present with primarily resectable disease. whereas, approximately $60 \%$ of patients are affected by metastatic disease [4]

Today, according to international guidelines, distant metastases (including non regional lymph nodes) and vascular infiltration are absolute contraindication to surgery [5]. Surgical resection of PDAC with synchronous distant metastases is not indicated as the average survival time appears equivalent to that of chemotherapy alone [6].

In the past, in the absence of active primary chemotherapy regimens, many surgeons attempted to resect liver or lymph node metastasis in a single operation or in two different times after resection of the primary with detrimental results in terms of survival and quality of life [7].

Today, the use of polychemotherapy regimens increases the chemosensitivity and the rate of response to the disease. In a Phase III randomized study, the combination chemotherapy FOLFIRINOX gained a significant advantage in terms of progression free survival (PFS) (6.4 months versus 3.3 months; $p<0.0001)$ and overall survival (OS) (11.1 months versus 6.8 months, 1 y OS versus $48.4 \% 20.6 \% \mathrm{p}<0.0001$ ) compared to gemcitabine in patients with metastatic disease and age $\leq 70$ years [8]. Furthermore, another randomized phase III study of 861 patients with mPDAC previously untreated have shown that the combination gemcitabine-nabpaclitaxel can improve PFS (HR 0.69; $p<0.0001$ ) and OS (HR 0.72; p <0.0001) compared to gemcitabine alone [9].

A crucial issue related to the actual improvement achieved with these new regimens is represented by the occasional possibility to observe a radiological complete response of metastatic lesions in patients with synchronous primary tumor [10]. What could be the best therapeutic management of these patients? Could surgery represent an indication? Herein, we discuss the role of surgery in a long term metastatic PDAC survivor who presented a complete response of synchronous liver metastases after modified FOLFIRINOX regimen.

\section{Case report}

A Caucasian 64-year-old man suffering from hypertension and diabetes presented with an history of abdominal pain in the last two months. A computer tomography (CT) scan of abdomen revealed a lesion of $4 \mathrm{~cm}$ in diameter localized in the head of pancreas (Figure 1A) without a clear cleavage 
plane from the descending part of duodenum and an initial dilatation of intrahepatic bile duct. Multiple secondary liver lesions ranging from 1 to $2 \mathrm{~cm}$ with a diameter were concomitant (Figure 1B-D). Serum levels of CEA and Ca 19.9 were $721 \mathrm{ng} / \mathrm{mL}$ and $11.200 \mathrm{U} / \mathrm{mL}$, respectively. A fineneedle biopsy of both pancreatic and one liver lesions reported the diagnosis of malignant cells compatible with moderately differentiated PDAC. First line chemotherapy according to modified FOLFIRINOX regimen (oxaliplatin $85 \mathrm{mg} / \mathrm{m}^{2}$, irinotecan $180 \mathrm{mg} / \mathrm{m}^{2}$, folinic acid $400 \mathrm{mg} / \mathrm{m}^{2}$, continuous-44 hours infusion of $5 \mathrm{FU} 2400 \mathrm{mg} / \mathrm{m}^{2}$, every 2 weeks) was started. After 4 cycles a CT scan of abdomen documented a partial response of all target lesions according to RECIST 1.1 criteria [11]. Nevertheless, due to the appearance of a sub-occlusive clinical scenario associated with an acute bacterial pneumonia and a rapid decay of performance status, patient underwent an esophageal-gastric-duodenum endoscopy showing duodenal stenosis associated with severe gastric displacement. Therefore, a palliative gastro-entero-anastomosis was performed associated with a targeted antibiotic therapy for pneumonia. In the following 3 months only best-supportive care was initiated, in order to improve patient's health status. At that time, a new CT scan documented the increase of both pancreatic and liver lesions, serum tumor markers and obstructive jaundice with high total bilirubin levels $(15.7 \mathrm{mg} / \mathrm{dL})$. As a consequence, a biliary drainage was implanted with a rapid restoration of normal bilirubin values. In the next month, FOLFIRINOX regimen was resumed and 6 cycles were administered without significant toxicities and a progressive decline of CEA and Ca 19.9. A new CT scan showed dimensional stability of both hepatic and pancreatic lesions associated with a prevalence of necrotic areas (Figure 2 A-D). Simultaneously, a PET was negative. After one month, an exploratory laparotomy was performed. Intraoperative extemporaneous histological examination of two liver lesions was negative for malignancy. Thus, a duodenocephalopancreasectomy was performed. Microscopic examination reported the diagnosis of PDAC with negative surgical margins and 7 out of 25 metastatic pancreatic lymph nodes (ypT2N1). After 2 months, a CT scan of chest and abdomen show no secondary lesions. No systemic chemotherapy was administered in the following 6 months. Next, two consecutive CT scans identified no metastases, showing only 3 three stable sub-centimetric liver nodules. During this period, a slow progressive increase of tumor markers was documented.

At the third radiological evaluation after surgery, liver and lung relapse was observed (Figure 3 AD). The patient underwent to administration of other 20 cycles of mFOLFIRINOX with a 1 year progression free survival. The most frequent observed mFOLFIRINOX-related grade 1-2 adverse events were diarrea, stomatitis, and anemia. Occasionally, grade 3 neutropenia, anemia, diarrhea, and fatigue required dosage adaptions. After, a second line with gemcitabine was administred for 6 
months due to the increase of liver and lung metastases. Globally, the patient achieved an OS of 45 months.

\section{Patient Record}

Treatment courses and clinical features of this patient were collected at the National Cancer Institute "Giovanni Paolo II" of Bari, Italy. The patient signed an informed consent for the study which was approved by the local Ethical Committee ( ${ }^{\circ} 424$ of 2013).

\section{Discussion}

Liver metastases strongly impact PDAC outcome and embody an unmet clinical need target, representing one of the main morbidity and mortality factors in these patients. However, sometimes liver metastases are sensitive to chemotherapy treatment due to greater drug delivery than pancreatic tissue [12]. Unfortunately, the complete radiological response to liver metastases is not supported by high quality literature data able to drive the specialist trough a given clinicaljudgment-based therapeutic decision [13].

Indeed, only scanty evidences derived from case reports and retrospective analyses have been published to date [14]. Limitations of these studies are the retrospective methodology employed, the population heterogeneity, the difference between the surgery volume of the referral centers involved and the lack of homogeneity of the primary cytoreductive therapy utilized. Moreover, the concordance between experts in the field is also insufficient. Despite the available evidences do not support upfront synchronous resection of PDAC liver metastases, conversion surgery after optimal response to chemotherapy justifies a reasonable optimism for such integrated therapeutic window [14]. It is rational to include our case clinical course within the PDAC phenotype identified by Frigerio et al., in which the complete response obtained on liver metastases to a first line cytoreduction, might predict a favorable clinical outcome with a median overall survival (OS) of 56 months for 24 out of 535 subjects $(4,5 \%)$ bridged to surgery. The regimen employed was either FOLFIRINOX (66\%) or gemcitabine-based therapy (34\%) [15], leading to $88 \%$ of R0 resection and to $17 \%$ of patients gaining a complete pathological response. The mortality rate was $0 \%$. Furthermore, also primary tumor excision along with synchronous metastatic surgical resection for 23 patients out of 1147 (2\%) in optimal response after either FOLFIRINOX (61\%) or gemcitabinebased therapy (39\%), showed a median OS of 34.1 months [16]. In light of the aforementioned data further reports confirmed analogous clinical behavior [17].

Analogous reports are derived from Crippa et al. [18], who published the results of a retrospective bi-institutional study on the role of surgery in patients with liver metastatic PDAC with good 
performance status who underwent primary chemotherapy with subsequent radiological response and biochemistry. The study included 127 patients who underwent various chemotherapy schemes: gemcitabine-based (44\%); FOLFIRINOX (8\%); cisplatin, gemcitabine, capecitabine and epirubicin $(\mathrm{PEXG}) /$ capecitabine and docetaxel (PDXG)/epirubicin and fluorouracil (PEFG) (48\%). 56 patients (44\%) had a complete (7\%) or partial $(37 \%)$ metastasis radiological response. Surgical treatment was considered in patients with complete or partial radiological response and with normalization of CA 19.9 or reduction of CA $19.9>90 \%$ compared to the initial value. 11 patients $(8.5 \%)$ underwent surgical resection. Median OS was 11 months in the entire cohort and 15 months for patients with complete/partial response. In this subgroup, OS was significantly longer in patients undergoing surgical resection (median OS: 46 months versus 11 months; $\mathrm{p}<0.0001$ ). Authors identified as independent survival factors: multi-chemotherapy (HR: 0.512), surgical resection (HR: 0.360), $>5$ liver metastases at diagnosis (HR: 3.515), reduction of CA $19.9<50 \%$ compared to diagnosis (HR: 2.708). A retrospective analysis and a low number of patients undergoing surgical resection affect the study methodology. Nonetheless, the data obtained inspired further well-designed statistically powered clinical trial (i.e. ClinicalTrials.gov Identifier: NCT02892305; NCT00855634). Indeed, Crippa et al. highlighted the fundamental role of patient selection in driving the therapeutic strategy, taking into account risk factors, cytoreductive regimen employed, prognostic determinants such as the radiological and biochemical response [18].

Conversely, some Authors showed that synchronous pancreatic and liver metastases resection upfront did not result in improved survival compared to palliative treatment (mOS range of 6 months) and does not appear to be justified [19-20].

Other evidences reported a small increase in survival for resection of synchronous PDAC liver metastases with acceptable safety in highly selected patients [21-22]. Hackert et al. published the results of a single-center retrospective study in which postoperative complications and survival were evaluated in 62 patients with PDAC with synchronous liver metastases undergoing pancreatic and hepatic primary surgical resection. Patients suffered from limited liver disease (oligometastatic pancreatic cancer) and in 57 patients an atypical liver resection of one or two metastases was performed. About $10 \%$ of patients developed a clinically significant pancreatic fistula, $6.4 \%$ postoperative bleeding; $3.2 \%$ of patients underwent second-surgery and 30 -day mortality was $1.6 \%$. Median OS was 12.3 months and 5-year survival was $8.1 \%$. Limitations of this study consisted of retrospective analysis and the lack of complete data regarding the adjuvant treatment employed [23]. 
Therefore, according to current evidences it is reasonable to suggest that in patients with liver oligometastatic PDAC cancer, surgery upfront indication would necessitate prospective controlled clinical trials to support clinical decisions.

Conversely, surgical treatment can be considered in highly selected metastatic PDAC cases with stringent response to primary chemotherapy in clinical trials at reference centers. However, to date there are no selection criteria for primitive or liver metastasis resection of mPDAC.

Given the presented elements it would be of paramount importance to identify two orders of criteria aimed to proper tailor the combination approach to mPDAC: biologic predictors might foster a personalized therapeutic plan and imaging criteria, able to resolve the response criteria dilemma, hold the promise to dissect the potential cure rate of a given patient subgroup. In some carefully selected cases after primary chemotherapy, the objective response assessment by imaging and tumor-markers can orientate the surgery choice.

Our case report highlights an extraordinary and apparently unpredictable disease course, arising unsolved clinical and pre-clinical question. Given that the complete response of hepatic metastasis in PDAC constitutes a rare event, an extensive biologic investigation can help to deeper characterize the underlying unsolved biologic phenotype. The genomic landscape appears to be one of the major challenging factors driving tumor heterogeneity [24-25]. Both distant metastases [2627] nodal involvement [28-29] and drug resistance [30-32] have been correlated with peculiar molecular signatures in PDAC. Cancer omics and biological signatures are able to stratify tumors depending on the cancer cell phenotype and the tumor niche, able to educate a neoplastic-friendly microenvironment for both solid and hematological cancer [33-37]. Resolving the spatial and clonal cancer heterogeneity, might provide fundamental clues able to deeper characterize translational target and oncogenic drivers, providing novel theragnostic targets.

The paradigm learned from colorectal carcinoma represents a pragmatic integration between biological prognostic factors and progressive resolution of comprehensive surgical-medical approach of metastatic colon carcinoma [38-39]. The lesson from these evidences, drove expanded indication for surgery in metastatic neuroendocrine [40] and renal cell carcinoma [41]. On the other hand, current guidelines do not support surgical approach for PDAC in metastatic setting [5].

To our knowledge this is the first report of coexistence of prolonged chemotherapeutic exposure along with clinical favorable outcome for a metastatic PDAC patient. He complained several mFOLFIRINOX related adverse events; nonetheless, we were able to administer more than 30 treatment cycles. Safety profile was acceptable in terms of supportive treatment. This multidimensional management displays paramount relevance, taking into account the frequent 
correlation between the length of treatment and the appearance of AE, which sometimes could require hospitalization [42-43]. In frame of this thinking the need of optimal patient selection would prevent unnecessary and unethical treatment, bridging the gap of stratified approach dedicated to subjects harboring clinical and biological signatures that predict more favorable outcome when approached with combined strategies [44-45].

\section{Figure legends}

Figure 1 - Staging: radiological evaluation of primary pancreatic lesion (A) and liver metastasis (BC)

Figure 2 - Radiological evaluation before surgery: primary pancreatic lesion (A) and liver metastasis (B-C)

Figure 3 - Disease relapse: radiological evaluation of primary pancreatic lesion (A), liver (B), and lung metastasis (C-D)

\section{Conflict of interests}

The Authors declare the absence of conflict of interests.

\section{Acknowledgment}

Partially supported by the Apulian Regional Project "Medicina di Pecisione".

\section{References}

1. Ferlay, J.; Shin, H.R.; Bray, F.; et al. Estimates of worldwide burden of cancer in 2008: GLOBOCAN 2008. Int J Cancer 2010, 127, 2893-2917.

2. Siegel, R.L..; Miller, K.D.; Jemal, A. Cancer statistics, 2019. CA Cancer J Clin 2019, 69, 7-34.

3. Rahib, L.; Smith, B.D.; Aizenberg, R.; et al. Projecting cancer incidence and deaths to 2030: the unexpected burden of thyroid, liver, and pancreas cancers in the United States. Cancer Res 2014, 74, 2913-2921.

4. Hidalgo, M. Pancreatic cancer. N Engl J Med 2010, 362, 1605-17.

5. Khorana, A.A.; McKernin, S.E.; Berlin, J.; et al. Potentially Curable Pancreatic Adenocarcinoma: ASCO Clinical Practice Guideline Update. J Clin Oncol 2019, 37, 20 822088.

6. Gleisner, A.L.; Assumpcao, L.; Cameron, J.L.; et al. Is resection of periampullary or pancreatic adenocarcinoma with synchronous hepatic metastasis justified? Cancer 2007, 110, 2484-2492. 
7. Koninger, J.; Wente, M.N.; Muller-Stich, B.P.; et al. R2 resection in pancreatic cancer-does it make sense? Langenbecks Arch Surg 2008, 393, 929-934.

8. Conroy, T.; Desseigne, F.; Ychou, M.; et al. FOLFIRINOX versus gemcitabine for metastatic pancreatic cancer. $N$ Engl J Med 2011, 364, 1817-25.

9. Von Hoff, D.D.; Ervin, T.; Arena, F.P.; et al. Increased survival in pancreatic cancer with nabpaclitaxel plus gemcitabine. N Engl J Med 2013, 369, 1691-703.

10. Zannini, N.; Lombardi, R.; Masetti, M.; et al. Surgery for isolated liver metastases from pancreatic cancer. Updates Surg 2015, 67, 19-25

11. Eisenhauer, E.A.; Therasse, P.; Bogaerts, J.; et al. New response evaluation criteria in solid tumours: Revised RECIST guideline (version 1.1). Eur J Cancer 2009, 45, 228-47.

12. Bahrami, A; Khazaei, M.; Bagherieh, F.; et al. Targeting stroma in pancreatic cancer: Promises and failures of targeted therapies. J Cell Physiol 2017, 232, 2931-2937.

13. Vaccaro, V.; Sperduti, I.; Vari, S.; et al. Metastatic pancreatic cancer: is there a light at the end of the tunnel? World J Gastroenterol 2015, 21, 4788-801.

14. Sakaguchi, T.; Valente, R.; Tanaka, K.; et al. Surgical treatment of metastatic pancreatic ductal adenocarcinoma: a review of current literature. Pancreatology 2019, 672-680.

15. Frigerio, I.; Regi, P.; Giardino, A.; et al. Downstaging in Stage IV Pancreatic Cancer: A New Population Eligible for Surgery? Ann Surg Oncol 2017, 24, 2397-2403.

16. Wright, G.P.; Poruk, K.E.; Zenati, M.S.; et al. Primary Tumor Resection Following Favorable Response to Systemic Chemotherapy in Stage IV Pancreatic Adenocarcinoma with Synchronous Metastases: a Bi-institutional Analysis. J Gastrointest Surg 2016, 20, 1830-1835.

17. Satoi, S.; Yamaue, H.; Kato, K.; et al. Role of adjuvant surgery for patients with initially unresectable pancreatic cancer with a long-term favorable response to non-surgical to nonsurgical anti-cancer treatments: results of a project study for pancreatic surgery by the Japanese Society of Hepato-Biliary-Pancreatic Surgery. J Hepatob Pancreat Sci 2013, 20, 590-600.

18. Crippa, S.; Bittoni, A.; Sebastiani, E., et al. Is there a role for surgical resection in patients with pancreatic cancer with liver metastases responding to chemotherapy? Eur J Surg Oncol 2016, 42, 1533-9.

19. Gleisner, A.L.; Assumpcao, L.; Cameron, J.L.; et al. Is resection of periampullary or pancreatic adenocarcinoma with synchronous hepatic metastasis justified? Cancer: Interdisciplinary International Journal of the American Cancer Society 2007, 110, 2484-2492.

20. Takada, T.; Yasuda, H.; Amano, H.; et al. Simultaneous hepatic resection with pancreatoduodenectomy for metastatic pancreatic head carcinoma: does it improve survival? Hepatogastroenterology 1997, 44, 567- 573. 
21. Shrikhande, S.V.; Kleeff, J.; Reiser, C.; et al. Pancreatic resection for M1 pancreatic ductal adenocarcinoma. Annals of Surgical Oncology 2007, 14, 118-127.

22. Seelig, S.K.; Burkert, B.; Chromik, A.M.; et al. Pancreatic resections for advanced M1pancreatic carcinoma: the value of synchronous metastasectomy. HPB surgery 2010, 2010.

23. Hackert, T.; Niesen, W.; Hinz, U.; et al. Radical surgery of oligometastatic pancreatic cancer. Eur J Surg Oncol 2017, 43, 358-363.

24. Bailey, P.; Chang, D.K.; Nones, K.; et al. Genomic analyses identify molecular subtypes of pancreatic cancer. Nature 2016, 531, 47-52.

25. Gnoni, A.; Licchetta, A.; Scarpa, A.; et al. Carcinogenesis of pancreatic adenocarcinoma: precursor lesions. Int J Mol Sci 2013, 14, 19731-62.

26. Valastyan, S.; Weinberg, R.A. Tumor metastasis: molecular insights and evolving paradigms. Cell 2011, 147, 275-92.

27. Khan, M.A.; Azim, S.; Haseeb Zubair, H.; et al. Molecular Drivers of Pancreatic Cancer Pathogenesis: Looking Inward to Move Forward. Int J Mol Sci 2017, 18, 779.

28. Xiao, Z.; Luo, G.; Liu, C.; et al. Molecular Mechanism Underlying Lymphatic Metastasis in Pancreatic Cancer. Biomed Res Int 2014, 2014: 925845.

29. Argentiero, A.; De Summa, S.; Di Fonte, R.; et al. Gene Expression Comparison between the Lymph Node-Positive and -Negative Reveals a Peculiar Immune Microenvironment Signature and a Theranostic Role for WNT Targeting in Pancreatic Ductal Adenocarcinoma: A Pilot Study. Cancers (Basel) 2019, 11, pii: E942.

30. Collisson, E.A.; Sadanandam, A.; Olson, P.; et al. Subtypes of pancreatic ductal adenocarcinoma and their differing responses to therapy. Nat Med 2011, 17, 500-503.

31. Swayden, M.; Iovanna, J.; Soubeyran, P. Pancreatic cancer chemo-resistance is driven by tumor phenotype rather than tumor genotype. Heliyon 2018; 4, e01055.

32. Porcelli, L.; Iacobazzi, R.M.; Di Fonte, R.; et al. CAFs and TGF- $\beta$ Signaling Activation by Mast Cells Contribute to Resistance to Gemcitabine/Nabpaclitaxel in Pancreatic Cancer. Cancers (Basel) 2019, 11, pii: E330.

33. Hanahan, D.; Weinberg, R.A. Hallmarks of cancer: the next generation. Cell 2011; 144, 646-74.

34. Karamitopoulou, E. Tumour microenvironment of pancreatic cancer: immune landscape is dictated by molecular and histopathological features. British journal of cancer 2019.

35. Harjunpää, H.; Llort Asens, M.; Guenther, C.; et al. Cell Adhesion Molecules and Their Roles and Regulation in the Immune and Tumor Microenvironment. Front Immunol 2019; 10:1078.

36. Solimando, A.G.; Brandl, A.; Mattenheimer, K.; et al. JAM-A as a prognostic factor and new therapeutic target in multiple myeloma. Leukemia 2018; 32, 736-743. 
37. Rudelius. M.; Rosenfeldt, M.T.; Leich, E.; et al. Inhibition of focal adhesion kinase overcomes resistance of mantle cell lymphoma to ibrutinib in the bone marrow microenvironment. Haematologica 2018, 103, 116-125.

38. Poultsides, G.A.; Schulick, R.D.; Pawlik, T.M. Hepatic resection for colorectal metastases: the impact of surgical margin status on outcome. HPB (Oxford) 2010, 12, 43-49.

39. Santini, D.; Spoto, C.; Loupakis, F.; et al. High concordance of BRAF status between primary colorectal tumors and related metastatic sites: implications for clinical practice. Ann Oncol 2010, $21,1565$.

40. Mayo, S.C.; de Jong, M.C.; Pulitano, C.; et al. Surgical management of hepatic neuroendocrine tumor metastasis: results from an international multi-institutional analysis. Ann Surg Oncol 2010, 17, 3129-3136.

41. Kavolius, J.P.; Mastorakos, D.P.; Pavlovich, C.; Russo, P.; Burt, M.E.; Brady, M.S. Resection of metastatic renal cell carcinoma. J Clin Oncol 1998, 16, 2261-2266.

42. Numico, G.; Longo, V.; Courthod, G.; et al. Cancer survivorship: long-term side-effects of anticancer treatments of gastrointestinal cancer. Curr Opin Oncol 2015, 27, 351-7.

43. Numico, G.; Cristofano, A.; Mozzicafreddo, A.; et al. Hospital admission of cancer patients: Avoidable practice or necessary care? PLoS One 2015, 10: e0120827

44. McGuigan, A.; Kelly, P.; Turkington, R.C.; Jones, C.; Coleman, H.G.; McCain, R.S. Pancreatic cancer: A review of clinical diagnosis, epidemiology, treatment and outcomes. World $J$ Gastroenterol 2018; 24, 4846-4861.

45. Porcelli, L.; Quatrale, A.E.; Mantuano, P.; et al. Optimize radiochemotherapy in pancreatic cancer: PARP inhibitors a new therapeutic opportunity. Mol Oncol 2013; 7, 308-22. 

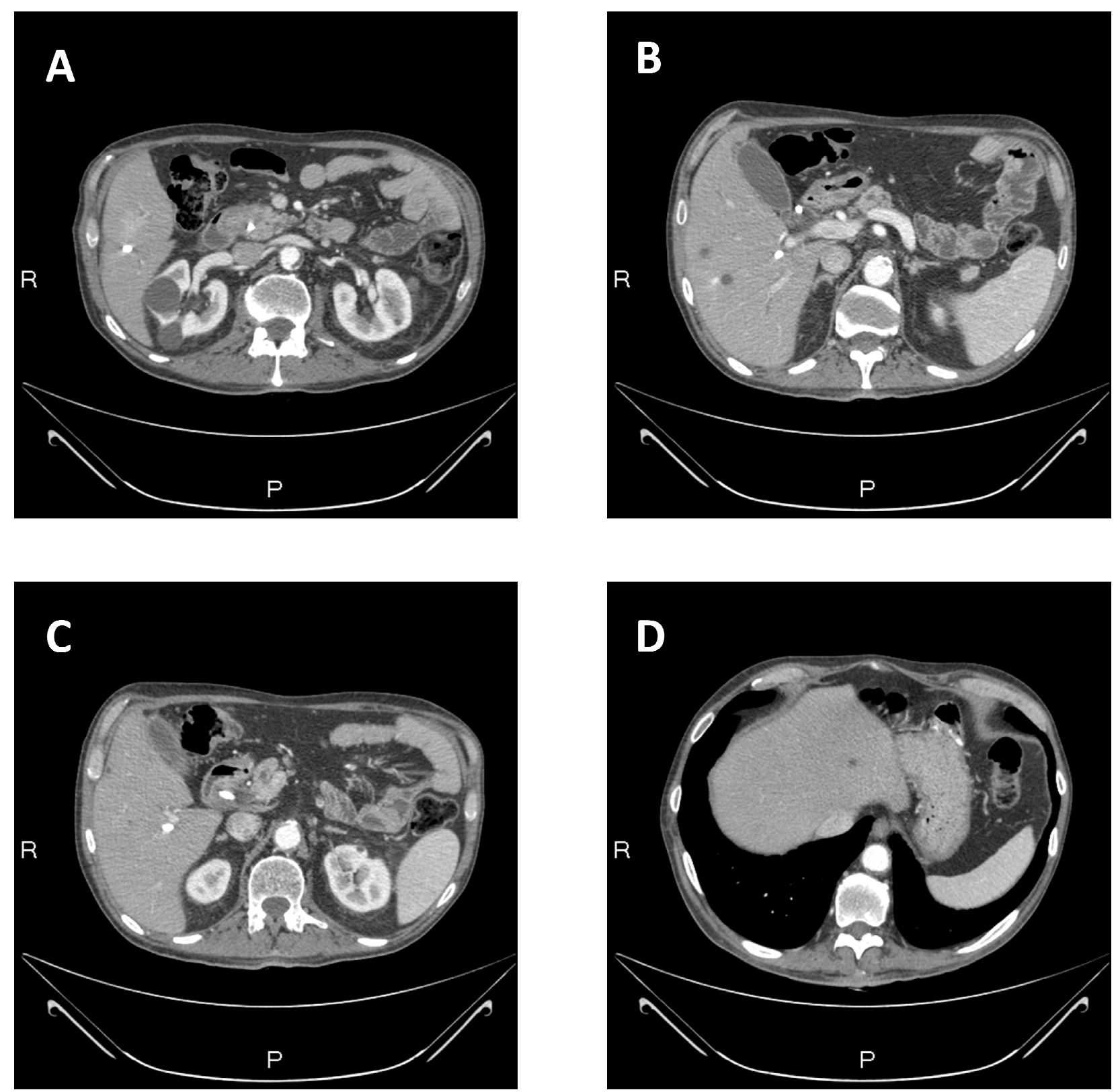

Figure 1 - Staging: radiological evaluation of primary pancreatic lesion (A) and liver metastasis (B-C) 

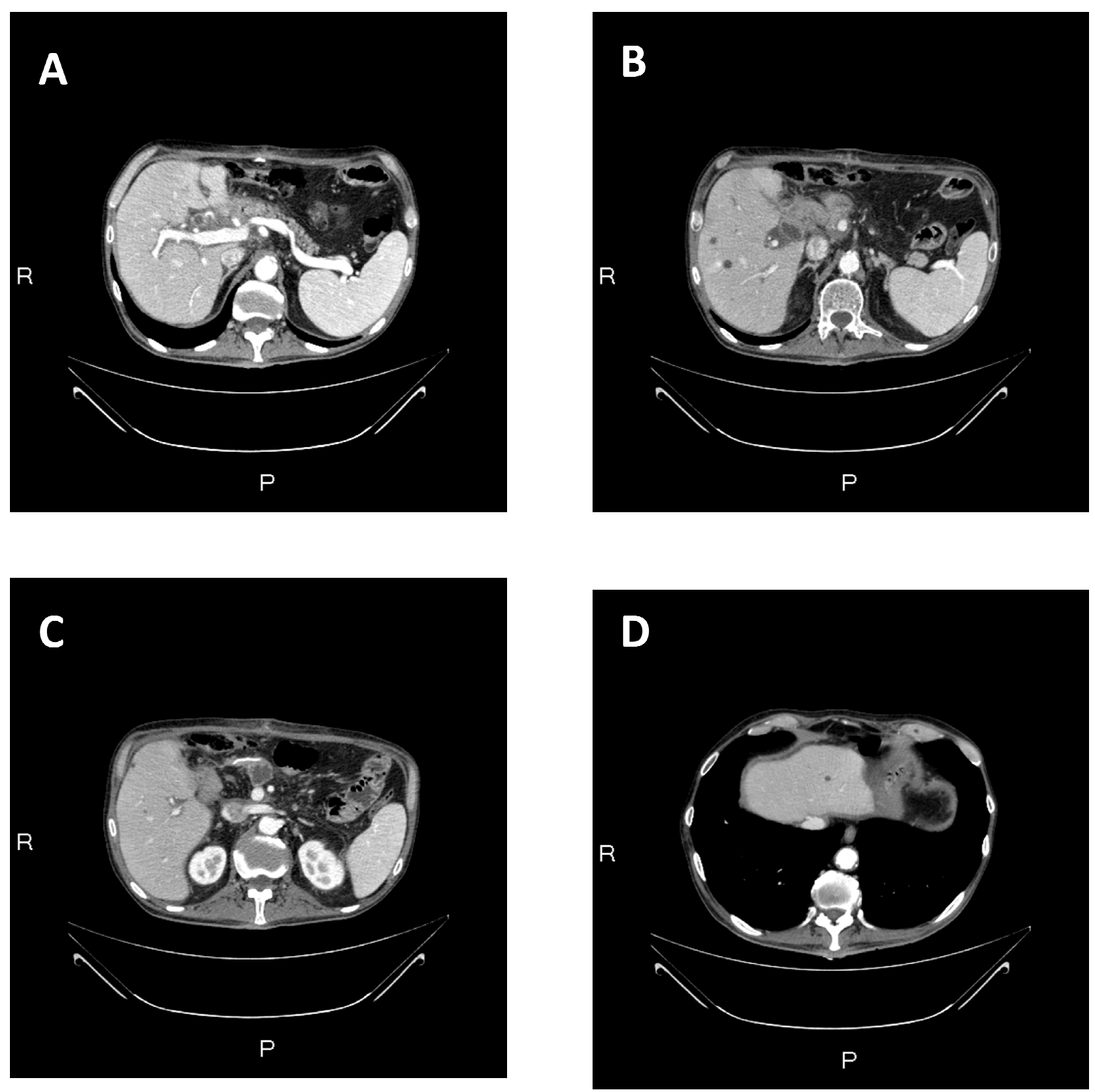

Figure 2 - Radiological evaluation before surgery: primary pancreatic lesion (A) and liver metastasis (B-D) 

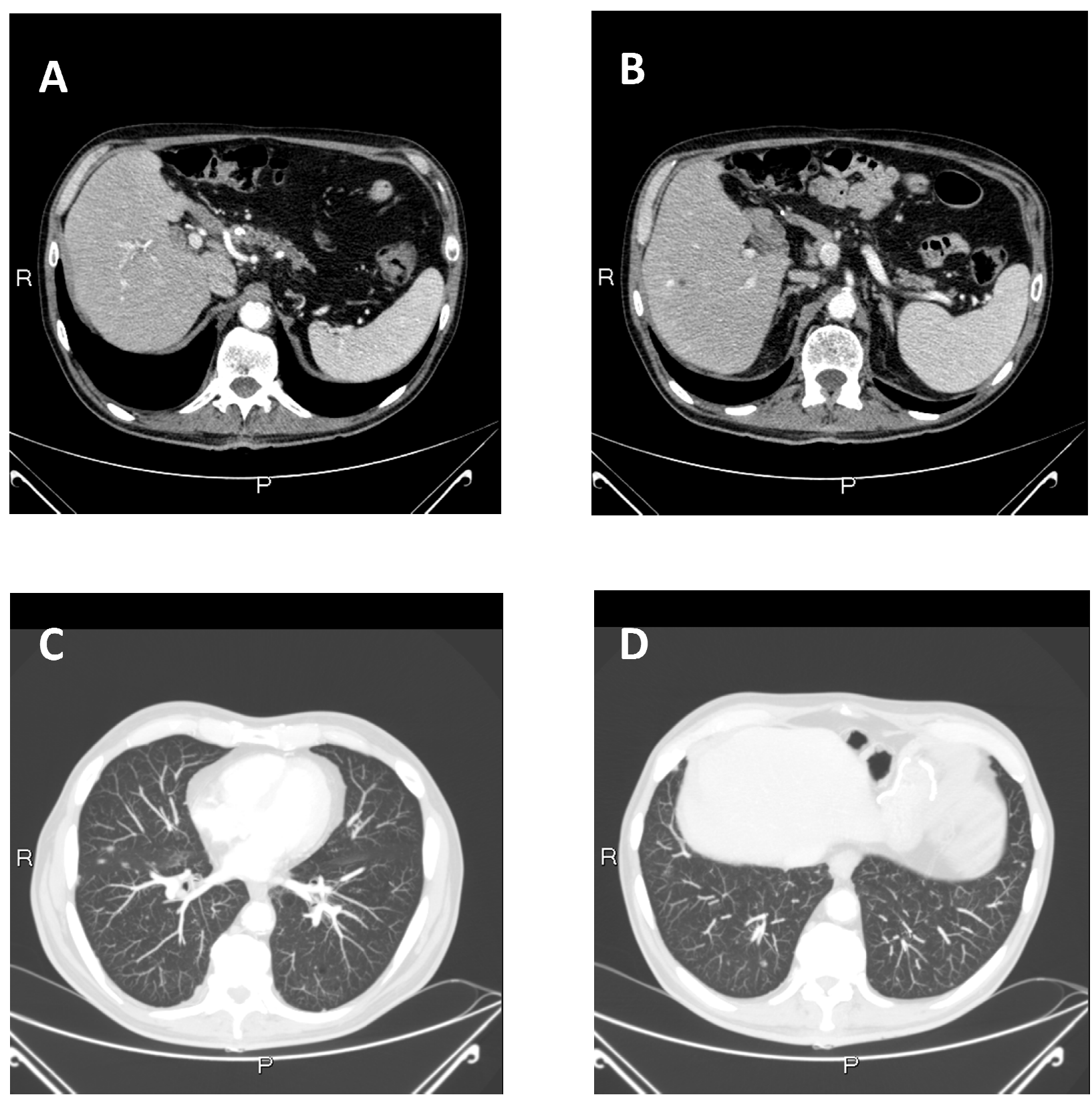

Figure 3 - Disease relapse: radiological evaluation of primary pancreatic lesion (A), liver (B), and lung metastasis (C-D) 